\section{Review Article}

\section{Corresponding Author}

Alexander J. Butler

(D) https://orcid.org/0000-0003-4135-7391

Department of Orthopaedic Surgery,

University of Miami Hospital, 1400 NW

12th Ave, Miami, FL 33136, USA

Tel: +1-201-585-1315

Fax: +1-305-324-7658

E-mail: alexander.butler@jhsmiami.org

Received: February 4, 2019

Revised: February 26, 2019

Accepted: March 4, 2019

\title{
Endoscopic Lumbar Surgery: The State of the Art in 2019
}

\author{
Alexander J. Butler', Milad Alam², Kevin Wiley ${ }^{2}$, Alexander Ghasem, \\ Augustus J. Rush III' ${ }^{1}$, Jeffrey C. Wang ${ }^{2}$ \\ ${ }^{1}$ Department of Orthopaedic Surgery, University of Miami Hospital, Miami, FL, USA \\ ${ }^{2}$ USC Spine Center, Los Angeles, CA, USA
}

This study was aimed to provide a brief historical perspective to facilitate appreciation of current techniques, describe outcomes of endoscopic lumbar surgery relative to those of existing techniques, and identify topics in need of study and future directions for the field of endoscopic lumbar surgery. Using the PubMed database, a comprehensive search was conducted to identify peer-reviewed English language articles pertaining to endoscopic lumbar surgery. Lack of focus on pertinent techniques or lack of outcome measures constituted exclusion criteria. A majority of included articles were published from 2015-2019. A context with which to appreciate the application of endoscopic lumbar techniques is established. An abundance of case series and several recent comparison studies have documented the benefits and potential pitfalls of these methods in the past two decades. The advantages of endoscopic lumbar spine surgery are widely touted to include reduced perioperative morbidity, including blood loss, operative time and immediate postoperative recovery, minimal structural trauma resulting from surgery, generally positive patient report outcome scores and the potential to contain costs. Additional high-quality research assessing outcomes of endoscopic lumbar surgery are certainly needed and currently expected given the rapid expansion of the field in recent years.

Keywords: Endoscopic spine surgery, Discectomy, Lumbar spine surgery, Minimally invasive surgery, Technology, Endoscopy

\section{INTRODUCTION}

Widely regarded as the inception of the development of endoscopic techniques in the spine, in 1983 Kambin and Gellman ${ }^{1}$ introduced a technique for nonvisualized percutaneous needle nucleotomy for the management of symptomatic degenerative discs. This method made use of a Craig biopsy canula via a posterolateral approach. Hijikata ${ }^{2}$ described a similar percutaneous technique in 1975. These pioneers followed more than a decade later with respective case series demonstrating generally positive results. ${ }^{3}$ The critical technological leap was taken by Forst and Hausmann ${ }^{4}$ in 1983, introducing an endoscope through this approach for direct visualization of the disc space for decompression. In the years since, these relatively deliberate beginnings have given way to tremendous technological advancement that today allows for many methods of high-quality visu- alization and treatment of various spinal pathology using endoscopy. Modern endoscopic spine surgery can be employed for the treatment of primary and recurrent disc disease, spinal stenosis, spondylolisthesis and spinal instability.

Despite recent applications of endoscopic spine surgery in the cervical and thoracic spine, this technique has to date been most frequently employed to address lumbar pathology. In the lumbar spine, the oldest and most utilized approach is an intraforaminal or transforaminal technique which allows for both intradiscal and extradiscal access. Described initially in 1990, Kambin triangle is the anatomic space bound by the caudal vertebral body (base), the traversing nerve root (height) and exiting nerve root (hypotenuse) at a given neuroforamen (Fig. 1). The intradiscal technique, which requires docking of the scope in the targeted disc space, provides relative stability of the endoscope. The extradiscal technique, by definition, does not vio- 
late the disc space, but it is less stable and requires a skilled surgeon's complete control of the scope. The interlaminar approach may also be employed at L5-S1, due to its advantage in avoiding the iliac crest by entering more midline through the ligamentum flavum. ${ }^{6}$ More medially based pathology may also be more effectively addressed using the interlaminar method.

Most transforaminal endoscopic lumbar procedures require local anesthesia and minimal sedation. The patient may be placed

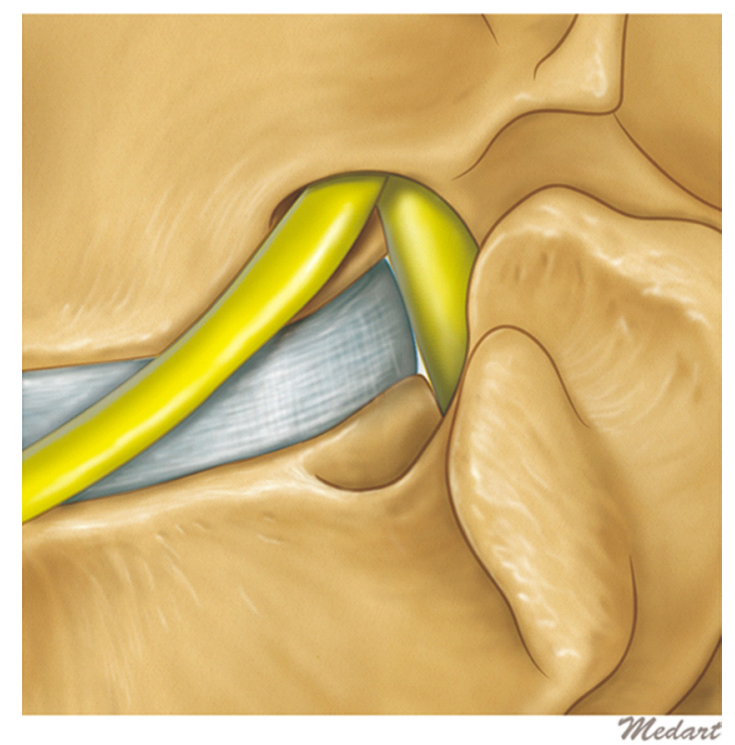

Fig. 1. Schematic depicting Kambin triangle, the anatomic space bound by the caudal vertebral body (base), the traversing nerve root (height), and exiting nerve root (hypotenuse) at a given neuroforamen. prone or in a lateral decubitus position on a standard frame depending on the pathology and planned approach. To utilize the transforaminal approach, a localization needle is advanced to Kambin triangle under fluoroscopic guidance in both planes. For the interlaminar approach, the needle is placed in the midline to approach the ligamentum flavum in the interlaminar space. After confirmation of the correct level and needle position, the cannula and endoscope are placed, and continuous saline irrigation allows for direct visualization of the surgical anatomy and pathology to be addressed (Fig. 2). A variety of angled scopes are typically available to maximize specific visualization. A uniportal technique requires instrumentation and the endoscope to be placed through the same portal, while both unilateral and bilateral biportal techniques have also been described..$^{7-9}$

\section{MATERIALS AND METHODS}

A comprehensive search of the PubMed database was performed utilizing various combinations of the following terms: "endoscopy," "endoscopic," "spine surgery," "lumbar surgery," "discectomy," "decompression," "foraminotmy," and "fusion." Articles published in peer-reviewed journals in the English language were included in an initial review of titles during which duplicates were excluded. Case series, case-control studies, retrospective cohort studies, randomized controlled trials and review articles were all included. A title and abstract review were then conducted; inclusion criteria included an explicit focus on surgery of the lumbar spine and endoscopic techniques as well
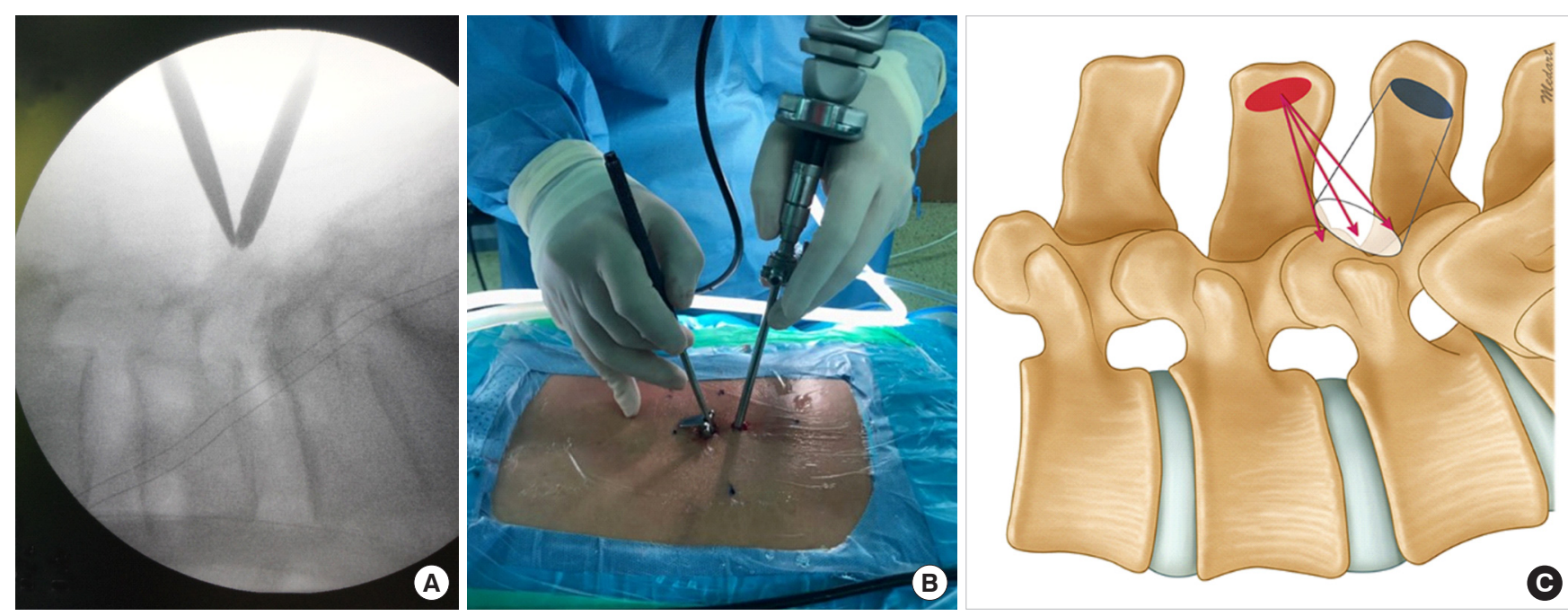

Fig. 2. Demonstration of instrumentation positioning and corresponding intraoperative fluoroscopic view (A) of the unilateral biportal technique. The surgeon holds the endoscope in his left hand (B) which corresponds to the blue viewing portal (C). Instruments in the surgeon's right hand (B) may be passed through the red working portal (C). 


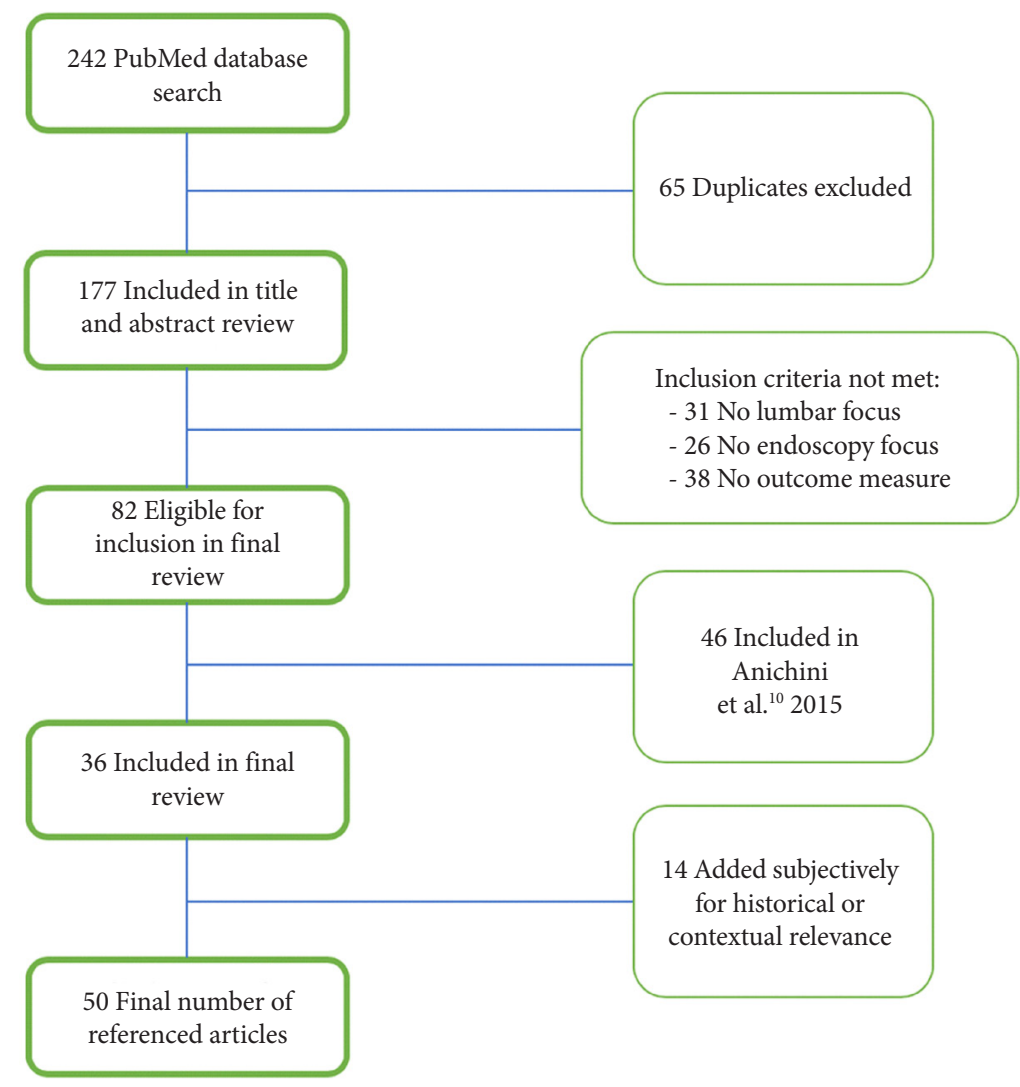

Fig. 3. Flowchart depicting article selection process accounting for inclusion in review of all referenced articles.

as use of objective outcome measures. Articles pertaining to endoscopic lumbar discectomy (ELD) that were included in a comprehensive systematic review published in 2015 were excluded. ${ }^{10}$ When applicable, reference lists of significant articles were cross-examined for additional articles appropriate for inclusion. An additional number of notable papers were included subjectively due to their historical or contextual relevance (Fig. 3).

\section{RESULTS}

\section{Lumbar Discectomy}

A descriptive review of the state of the literature regarding ELD published in 2015 identified a total of 51 case series on the topic published between 2009 and $2015 .{ }^{10}$ To varying extents between these series clinical results are described, with only 4 included comparisons to open techniques. The field has certainly benefited from additional such series in the several years since, however more significantly a welcome influx of higherquality data via additional comparative studies, meta-analyses and randomized controlled trials has been seen.

In a recent meta-analysis of comparison studies, Kim et al. ${ }^{11}$ found that ELD was superior to open lumbar discectomy (OLD) with regards to improvement in preoperative visual analogue scale (VAS) pain scores and Oswestry Disability Index (ODI) as well as total operative time. The postoperative hospital stay was also significantly longer in the OLD cohort by a difference of greater than 4 days. No differences in overall complication rate, recurrence rate of disc pathology or overall reoperation rate were noted. Of note, no randomized studies were included in this analysis.

Shi et al. ${ }^{12}$ recently published a similar meta-analysis of 18 heterogenous comparison studies consisting of 8 prospective studies with varying degrees of randomization and 10 retrospective studies comparing ELD to OLD. All included works were completed in Asia, with 17 from China. They found no differences in total operative time, improvement in preoperative ODI, VAS for leg pain, total complications, recurrence or incidence of durotomy. ELD was noted to be superior in operative blood loss, incision length, postoperative in bed time, length of hospital stay, and VAS for back pain. These authors determined that patients treated with OLD had a higher reoperation rate as well as less use of intraoperative fluoroscopy. Interestingly, du- 
ration of the operation was longer for ELD cases completed earlier in the study period, though improved to be significantly shorter when cases completed in 2018 or later were isolated. Overall procedural costs were generally higher for ELD though regional factors were cited as likely confounding.

An additional meta-analysis of 7 nonrandomized retrospective studies comparing ELD to OLD in the Korean population found no differences in VAS for leg pain, improvement in preoperative ODI, overall complication rate, recurrence rate, reoperation rate and total operative duration. ${ }^{13}$ ELD was superior in total hospital stay as well as parameters assessing return to work.

Alvi et al. ${ }^{14}$ published results of a systematic review evaluating 14 randomized, single center studies from from Asia, Europe, and the United States that included comparisons of OLD with minimally invasive techniques consisting of both tubular discectomy (TD) and ELD in the lumbar spine. When included, specific stratification to directly compare ELD to open techniques demonstrated a lower rate of overall complications and dural tears in ELD cohorts. TD and ELD collectively had a higher rate of disc herniation recurrence and overall reoperation rate, while no significant difference in any patient-reported outcome score was identified.

To the authors' knowledge, data from only one prospective, randomized controlled trial directly comparing outcomes between ELD and TD has been published to date. Preliminary results from a trial ongoing at the The Third Affiliated Hospital of Sun Yat-sen University, a large public university hospital in Guangzhou, China, were made available in $2018 .{ }^{15}$ One-year follow-up data did not demonstrate clear clinical superiority of either technique in 153 patients. All outcome measures, complication rates, incidence of recurrence and reoperation rates were similar in both groups. Interestingly, when stratified for location of pathology, ELD was superior with regards to improvement in preoperative ODI when treating far-lateral herniations though was inferior for para-median disease. This likely speaks to the advantages and limitations of the transforaminal approach that was widely used.

\section{Extraforaminal Decompression}

While the exploration of applications of endoscopic spine surgery in the lumbar spine has undoubtedly historically focused on discectomy for symptomatic herniation, other procedures have also been described. For the treatment of foraminal stenosis, focal decompression of affected nerve roots has been proposed as a valid alternative to the indirect decompression achieved with open laminectomy, foraminotomy and instru- mented fusion. These minimally invasive methods seek to avoid the morbidity associated with open fusion surgery as well as the potential disadvantages such as paraspinal muscle atrophy, adjacent segment disease and pseudoarthrosis. ${ }^{11}$ A cadaveric study published in 2015 demonstrated that fully endoscopic techniques can be effective in increasing foraminal height and area via decompression of extraforaminal structures including hypertrophic facet joint capsule, caudal pedicle, superior articular process and associated osteophytes. ${ }^{16}$ This served to provide mechanistic support for several existing case series describing collectively positive results. Ahn et al. ${ }^{17}$ have published several such series, the first of which in 2003 presented 12 patients with foraminal stenosis undergoing endoscopic foraminal decompression. A $83.3 \%$ of patients reported good to excellent results via the Macnab criteria at mean follow-up of 13 months. A subsequent series of patients with bony foraminal stenosis and corresponding radiculopathy were similarly treated with endoscopic foraminotomy with discectomy as indicated, with $81.8 \%$ reporting good to excellent results via the MacNab criteria at 2-year follow-up. ${ }^{18}$ A $93.9 \%$ of patients reported symptomatic improvement. Of note, all the procedures included in these series by Ahn et al. ${ }^{17,18}$ were completed with local anesthetic and light sedation, without any reported complications.

\section{Central Decompression}

The use of endoscopic techniques for the treatment of central lumbar stenosis has been explored due to the targeted advantages of preserving native spinal stability and paraspinal musculature while addressing focal areas of central stenosis. ${ }^{19}$ Endoscopic applications for this specific pathology may find their origin in a seminal paper by Khoo and Fessler ${ }^{20}$ in 2002 comparing 25 patients who had undergone their described technique of endoscopic-assisted laminotomy, notably not a full-endoscopic surgery, to another group of 25 patients having undergone traditional open laminotomy. Without differences in clinical outcome, endoscopic-assisted procedures had significantly less operative blood loss, shorter postoperative hospital stay and lower postoperative narcotic usage when compared to open. Many case series have described technical modifications, pitfalls and clinical results with generally positive patient-reported outcome scores for both endoscopic-assisted and fully endoscopic decompression surgery. ${ }^{8,21,22}$ A large-scale retrospective database study published in 2018 described significant superiority of endocopic-assisted, microendoscopic decompression versus open laminectomy with regards to total complication rate, surgical site infection, postoperative hospital stay and inci- 
dence of postoperative delirium. ${ }^{23}$ A total of 1536 matched pairs of patients were included in this analysis. With regards to fully endoscopic techniques, a 2017 retrospective series of 48 patients treated with fully endoscopic decompression for central lumbar stenosis documented a $96 \%$ rate of good-excellent outcome via the Macnab outcome grade. These results were reported in the setting of relatively low complications consisting of 3 dural tears; the significance of the learning curve for this technique and its impact on early technical fault was emphasized by the authors. ${ }^{24}$ An additional retrospective analysis of 213 patients treated with fully endoscopic laminotomy and flavectomy more recently reported significant improvements in patient-reported outcomes scores and a $0 \%$ reoperation rate for incomplete decompression with mean 26-month follow-up. ${ }^{25}$ The authors also cited a reduction in mean operation time by as much as two-thirds during the late stage of the learning curve upon implementing this technique. A randomized controlled trial including a total of 135 patients receiving fully endoscopic versus minimally invasive laminotomy found no significant differences in clinical outcome at 2-year follow-up. ${ }^{26}$ The endoscopic group had significantly fewer complications, shorter operative time and faster postoperative rehabilitation, though reoperation rate was similar between the 2 groups. Based on currently available literature, endoscopic laminotomy for lumbar spinal stenosis may be considered an acceptable surgical method once surgeons have demonstrated comfort with the learning curve.

\section{Lumbar Fusion}

An additional application of endoscopic techniques in the lumbar spine is that of fusion. Instrumented minimally invasive surgery (MIS) fusion techniques find their roots in Magerl's 1984 description of percutaneous pedicle screw placement for the purpose of external fixation. ${ }^{27}$ In 2001 Foley et al. ${ }^{28}$ took advantage of this method by contributing the development of a percutaneously inserted connecting rod to describe the first MIS internal fixation construct for the spine. In the interim, Leu and Hauser ${ }^{29}$ published a series in 1996 describing the development and early clinical results of a technique for percutaneous endoscopic lumbar fusion (PELF) with 12 weeks of postoperative pedicular external fixation. In a single-surgeon cohort of 37 patients undergoing this procedure, 30 progressed to stable bony union with mean 33- month follow-up. Minimal perioperative morbidity, low complication rate and generally positive clinical outcomes are described though poorly quantified.

As most modern applications of endoscopy for lumbar fusion take advantage of the capabilities of MIS fusion, worth mention- ing is the contemporaneous development of MIS fusion techniques without the use of endoscopy, most notably lumbar interbody fusion (LIF). First described by Harms and Rolinger in 1982, the transforaminal lumbar interbody fusion (TLIF) has since been popularized due to the advantages of greater indirect foraminal decompression and restoration of lumbar lordosis in the setting of reduced disruption of bony stability as well as perioperative morbidity. ${ }^{30-32}$ While one advantage of MIS-TLIF relative to open techniques is reduced paraspinal trauma, the clinical impact of morbidity resulting from tubular MIS approaches may be further reduced by the use of endoscopy. The inherent limitations of visualization with tubular methods have also been described. ${ }^{11,33,34}$

With this stage set, in 2013 Jacquot and Gastambide ${ }^{35}$ published results of their initial experience with endoscopic TLIF performed on 57 patients from 2004 to 2010. Despite describing short operative duration and a 100\% fusion rate, this widely cited article concludes with the recommendation that their surgical methods not be utilized in their current state due to a $36 \%$ rate of complications directly attributable to technical error. This established a relative burden of proof for literature on endoscopic fusion techniques to overcome. Since this paper's publication, several technological and technical advancements have been made with regards to endoscopic lumbar fusion utilizing both interbody and pedicle screw fixation, with several notable case series now available. Heo et al. ${ }^{33}$ describe a technique of endoscopy-assisted LIF using the biportal technique followed by percutaneous single level instrumentation. Sixty-nine patients with symptomatic, single level stenosis or instability treated in this manner had significant improvement in preoperative VAS and ODI scores at a mean of 14-month follow-up. Magnetic resonance imagings (MRIs) performed on postoperative day 2 demonstrated "optimal" decompression at the pathologic level in all patients. Complications described consisted of 2 dural tears and 2 epidural hematomas. Wu et al..$^{34}$ have presented initial results from 7 patients treated with a similar technique with minimum 2-year follow-up, all with improvement in ODI, VAS, and SF36 Physical and Mental Component Surveys. Endoscopic single level TLIF with percutaneous pedicle screws under conscious sedation has also been described. ${ }^{36}$ Ten patients treated for single level loss of disc height, 6 with grade I spondylolisthesis, reported improvements in patient reported outcome measures with radiographic union at 1 year. The authors commented on reduced operative time, blood loss and hospital stay. In addition to endoscopic fusion using a posterior transforaminal approach, a lateral route has also been described in a small recent series in 
14 patients with either central or foraminal disc herniations. ${ }^{37}$ Clinical outcomes were generally good, and postoperative MRI confirmed adequate decompression in all cases, indicating this approach as a reasonable option for direct neural decompression.

To the authors' knowledge only 1 comparative study has been published to date evaluating outcomes of PELF versus open or MIS techniques. ${ }^{38} 56$ nonrandomized patients were treated at a single center with either open posterior lumbar interbody fusion (PLIF) or endoscopic assisted tubular PLIF. Open procedures had a shorter total operative time, though the endoscopic group was characterized by less blood loss, shorter postoperative hospitalization and less immediate postoperative back pain likely reflecting the less traumatic surgical approach. Authors of each of these series include mention of the significant promise in reducing blood loss, perioperative morbidity, hospital stay and immediate functional status relative to open and MIS techniques.

Relative to ELD, data-supported adoption of endoscopic fusion techniques seems to remain in its infancy. Within the last year, several articles from China comparing endoscopic discectomy to MIS TLIF have been published, with inconclusive results. ${ }^{38-40}$ Future comparative and randomized studies evaluating outcomes of endoscopic versus MIS or open lumbar fusions are expected.

\section{DISCUSSION}

With origins in the early development of MIS techniques, and taking advantage of modern technology, significant innovations in the field of endoscopic spine surgery have been made in recent years. The primary advantages of endoscopic lumbar surgery lie in minimizing the structural and clinical impact of the surgical approach. With a smaller surgical incision and approach come a lower risk of infection, less operative blood loss and less overall soft tissue trauma. Specific to spine surgery, maintenance of structural integrity and innervation of paraspinal musculature as well as less epidural scarring are thought to facilitate postoperative motion, strength and subsequent improved functional outcome and patient satisfaction. These factors in conjunction with reduced operative time and need for general anesthesia are also likely to lead to reduced postoperative hospital stay which also has implications on cost and value of care provided. Formal investigations of total hospital cost comparing endoscopic versus MIS or open lumbar surgery are lacking, though no benefit has been demonstrated to date. ${ }^{12}$ As systemic infrastructure grows with implementation of these techniques, further research may demonstrate the impact of the aforementioned specific clinical advantages on cost. Additionally, current global obstacles facing the possibility of making large-scale cost savings via outpatient spine surgery may soon be addressed by the reduced perioperative morbidity that endoscopy can provide. $^{41}$

A consistently described disadvantage of endoscopy lumbar surgery is that of the steep and potentially dangerous learning curve that accompanies the technical demand of its application. As previously mentioned, Shi et al. ${ }^{12}$ described a reduction in overall operative duration in sequential years of PELD at multiple centers, possibly implicating operative efficiency as surgeon's skill evolved. Multiple series and comparison studies have described either a higher incidence of overall complications or longer operative time during the early phases of the respective study period, when quantified. ${ }^{42-44}$ Operative duration has been described as significantly longer in the first 10-14 procedures completed by single surgeons, with relative uniformity after this initial learning period. ${ }^{9,45}$ Specific factors requiring improved surgeon exposure and comfort level for optimal use include a new surgical approach, manipulation of endoscopic equipment and visuospatial orientation when viewing the endoscopic image. While a learning curve must certainly be respected by any surgeon adopting endoscopic techniques, current sentiment supports the general feasibility of safe and successful integration into the erudite spine surgeon's practice.

Albeit with some heterogeneity, currently available case series and comparative studies appear to support a lower overall complication rate with endoscopic procedures relative to their MIS or open counterparts. The most commonly reported complications of endoscopic lumbar surgery include dural tear, infection, epidural hematoma. ${ }^{10,12,23,46,47}$ Additional data is of course needed prior to making any definitive determination regarding the promise of reduced complications through the use of endoscopy. The only randomized study to date directly comparing and endoscopic lumbar procedure to his MIS counterpart has not found improved complication rate at preliminary 1-year followup. ${ }^{15}$

Interestingly, there are several complications specific to endoscopic spine surgery worth noting. Postoperative headache as well as several rare cases of postoperative seizure following prodromal neck pain have been described. ${ }^{48}$ Thought to be secondary to increased epidural pressure from the endoscopic irrigation system, all such reported complications resolved with conservative management. The suggestion of increased risk for durotomy when endoscopic central decompression has been 
made, possibly due to new visuospatial or tactile demands when using the endoscope. ${ }^{21}$ Contrastingly, a more recent article noted a reduced rate of dural tears in endoscopic versus MIS and open techniques. ${ }^{14}$ Radicular pain and paresthesia corresponding to the exiting nerve root at the operative level has also been described. Proposed mechanisms are direct irritation from the canula, indirect pressure from the irrigation system, effect of local anesthetic used during the procedure. ${ }^{47,49}$

\section{CONCLUSION}

The field of spine surgery recently has borne witness to the explosive growth of literature describing the applications of endoscopy for treating lumbar pathology. While posing a potential technical challenge for surgeons adopting these techniques, the promise of reduced perioperative morbidity in the setting of positive outcomes has propelled endoscopic spine surgery to the forefront of current innovation. As the development of surgical techniques and devices continues, further high-quality data is anticipated to assess its ultimate superiority over existing methods.

\section{CONFLICT OF INTEREST}

The authors have nothing to disclose.

\section{REFERENCES}

1. Kambin P, Gellman H. Percutaneous lateral discectomy of the lumbar spine: a preliminary report. Clin Orthop Relat Res 1983;174:127-32.

2. Hijikata S, Yamagishi M, Nakayaka T, et al. Percutaneous nucleotomy: a new treatment method for lumbar dise herniation (in Japanese). J Toden Hosp 1975;5:39-45.

3. Hijikata S. Percutaneous nucleotomy. A new concept technique and 12 years' experience. Clin Orthop Relat Res 1989; (238):9-23.

4. Forst R, Hausmann B. Nucleoscopy--a new examination technique. Arch Orthop Trauma Surg 1983;101:219-21.

5. Kambin P. Arthroscopic microdiscectomy: minimal intervention spinal surgery. Baltimore (MD): Urban \& Schwarzenberg; 1990.

6. Ruetten S, Komp M, Godolias G. A New full-endoscopic technique for the interlaminar operation of lumbar disc herniations using 6-mm endoscopes: prospective 2-year results of 331 patients. Minim Invasive Neurosurg 2006;49:80-7.

7. Kambin P, Gennarelli T, Hermantin F. Minimally invasive techniques in spinal surgery: current practice. Neurosurg Focus 1998;4:Article 8.

8. Hwa Eum J, Hwa Heo D, Son SK, et al. Percutaneous biportal endoscopic decompression for lumbar spinal stenosis: a technical note and preliminary clinical results. J Neurosurg Spine 2016;24:602-7.

9. Choi CM, Chung JT, Lee SJ, et al. How I do it? Biportal endoscopic spinal surgery (BESS) for treatment of lumbar spinal stenosis. Acta Neurochir (Wien) 2016;158:459-63.

10. Anichini G, Landi A, Caporlingua F, et al. Lumbar endoscopic microdiscectomy: where are we now? An updated literature review focused on clinical outcome, complications, and rate of recurrence. Biomed Res Int 2015;2015:417801.

11. Kim M, Lee S, Kim HS, et al. A comparison of percutaneous endoscopic lumbar discectomy and open lumbar microdiscectomy for lumbar disc herniation in the korean: a metaanalysis. Biomed Res Int 2018;2018:9073460.

12. Shi R, Wang F, Hong X, et al. Comparison of percutaneous endoscopic lumbar discectomy versus microendoscopic discectomy for the treatment of lumbar disc herniation: a meta-analysis. Int Orthop 2018 Dec 13 [Epub]. https://doi. org/10.1007/s00264-018-4253-8.

13. Qin R, Liu B, Hao J, et al. Percutaneous endoscopic lumbar discectomy versus posterior open lumbar microdiscectomy for the treatment of symptomatic lumbar disc herniation: a systemic review and meta-analysis. World Neurosurg 2018; 120:352-62.

14. Alvi MA, Kerezoudis P, Wahood W, et al. Operative approaches for lumbar disc herniation: a systematic review and multiple treatment meta-analysis of conventional and minimally invasive surgeries. World Neurosurg 2018;114:391407.e2.

15. Chen Z, Zhang L, Dong J, et al. Percutaneous transforaminal endoscopic discectomy compared with microendoscopic discectomy for lumbar disc herniation: 1-year results of an ongoing randomized controlled trial. J Neurosurg Spine 2018;28:300-10.

16. Evins AI, Banu MA, Njoku I Jr, et al. Endoscopic lumbar foraminotomy. J Clin Neurosci 2015;22:730-4.

17. Ahn Y, Lee SH, Park WM, et al. Posterolateral percutaneous endoscopic lumbar foraminotomy for L5-S1 foraminal or lateral exit zone stenosis. Technical note. J Neurosurg 2003; 99(3 Suppl):320-3.

18. Ahn Y, Oh HK, Kim H, et al. Percutaneous endoscopic lumbar foraminotomy: an advanced surgical technique and clinical outcomes. Neurosurgery 2014;75:124-33. 
19. Choi DJ, Choi CM, Jung JT, et al. Learning curve associated with complications in biportal endoscopic spinal surgery: challenges and strategies. Asian Spine J 2016;10:624-9.

20. Khoo LT, Fessler RG. Microendoscopic decompressive laminotomy for the treatment of lumbar stenosis. Neurosurgery 2002;51(5 Suppl):S146-54.

21. Pao JL, Chen WC, Chen PQ. Clinical outcomes of microendoscopic decompressive laminotomy for degenerative lumbar spinal stenosis. Eur Spine J 2009;18:672-8.

22. Castro-Menéndez M, Bravo-Ricoy JA, Casal-Moro R, et al. Midterm outcome after microendoscopic decompressive laminotomy for lumbar spinal stenosis: 4-year prospective study. Neurosurgery 2009;65:100-10.

23. Oichi T, Oshima Y, Chikuda H, et al. In-hospital complication rate following microendoscopic versus open lumbar laminectomy: a propensity score-matched analysis. Spine J 2018;18:1815-21.

24. Kim HS, Paudel B, Jang JS, et al. Percutaneous full endoscopic bilateral lumbar decompression of spinal stenosis through uniportal-contralateral approach: techniques and preliminary results. World Neurosurg 2017;103:201-9.

25. Lee CW, Yoon KJ, Jun JH. Percutaneous endoscopic laminotomy with flavectomy by uniportal, unilateral approach for the lumbar canal or lateral recess stenosis. World Neurosurg 2018;113:e129-37.

26. Komp M, Hahn P, Oezdemir S, et al. Bilateral spinal decompression of lumbar central stenosis with the full-endoscopic interlaminar versus microsurgical laminotomy technique: a prospective, randomized, controlled study. Pain Physician 2015;18:61-70.

27. Magerl FP. Stabilization of the lower thoracic and lumbar spine with external skeletal fixation. Clin Orthop Relat Res 1984;(189):125-41.

28. Foley KT, Gupta SK, Justis JR, et al. Percutaneous pedicle screw fixation of the lumbar spine. Neurosurg Focus 2001; 10:E10.

29. Leu HF, Hauser RK. Percutaneous endoscopic lumbar spine fusion. Neurosurg Clin N Am 1996;7:107-17.

30. Harms J, Rolinger H. A one-stager procedure in operative treatment of spondylolistheses: dorsal traction-reposition and anterior fusion (author's transl). Z Orthop Ihre Grenzgeb 1982;120:343-7.

31. Humphreys SC, Hodges SD, Patwardhan AG, et al. Comparison of posterior and transforaminal approaches to lumbar interbody fusion. Spine (Phila Pa 1976) 2001;26:567-71.

32. Hee HT, Castro FP Jr, Majd ME, et al. Anterior/posterior lumbar fusion versus transforaminal lumbar interbody fusion: analysis of complications and predictive factors. J Spinal Disord 2001;14:533-40.

33. Heo DH, Son SK, Eum JH, et al. Fully endoscopic lumbar interbody fusion using a percutaneous unilateral biportal endoscopic technique: technical note and preliminary clinical results. Neurosurg Focus 2017;43:E8.

34. Wu J, Liu H, Ao S, et al. Percutaneous endoscopic lumbar interbody fusion: technical note and preliminary clinical experience with 2-year follow-up. Biomed Res Int 2018;2018: 5806037.

35. Jacquot F, Gastambide D. Percutaneous endoscopic transforaminal lumbar interbody fusion: is it worth it? Int Orthop 2013;37:1507-10.

36. Wang MY, Grossman J. Endoscopic minimally invasive transforaminal interbody fusion without general anesthesia: initial clinical experience with 1-year follow-up. Neurosurg Focus 2016;40:E13.

37. Heo DH, Kim JS. Clinical and radiological outcomes of spinal endoscopic discectomy-assisted oblique lumbar interbody fusion: preliminary results. Neurosurg Focus 2017;43:E13.

38. Liu C, Zhou Y. Percutaneous endoscopic lumbar discectomy and minimally invasive transforaminal lumbar interbody fusion for massive lumbar disc herniation. Clin Neurol Neurosurg 2019;176:19-24.

39. Liu C, Zhou Y. Comparison between percutaneous endoscopic lumbar discectomy and minimally invasive transforaminal lumbar interbody fusion for lumbar disc herniation with biradicular symptoms. World Neurosurg 2018;120:e72-9.

40. Li YW, Wang HJ, Wang YS, et al. Comparison of percutaneous endoscopic lumbar discectomy versus transforaminal lumbar interbody fusion in treating upper lumbar disc herniation. Zhonghua Yi Xue Za Zhi 2018;98:113-6.

41. Pendharkar AV, Shahin MN, Ho AL, et al. Outpatient spine surgery: defining the outcomes, value, and barriers to implementation. Neurosurg Focus 2018;44:E11.

42. Marappan K, Jothi R, Paul Raj S. Microendoscopic discectomy (MED) for lumbar disc herniation: comparison of learning curve of the surgery and outcome with other established case studies. J Spine Surg 2018;4:630-7.

43. Nowitzke AM. Assessment of the learning curve for lumbar microendoscopic discectomy. Neurosurgery 2005;56:75562.

44. Soliman HM. Irrigation endoscopic discectomy: a novel percutaneous approach for lumbar disc prolapse. Eur Spine J 2013;22:1037-44 
45. Ahn SS, Kim SH, Kim DW, et al. Comparison of outcomes of percutaneous endoscopic lumbar discectomy and open lumbar microdiscectomy for young adults: a retrospective matched cohort study. World Neurosurg 2016;86:250-8.

46. Kim JE, Choi DJ. Unilateral biportal endoscopic spinal surgery using a $30^{\circ}$ arthroscope for L5-S1 foraminal decompression. Clin Orthop Surg 2018;10:508-12.

47. Sairyo K, Matsuura T, Higashino K, et al. Surgery related complications in percutaneous endoscopic lumbar discecto- my under local anesthesia. J Med Invest 2014;61:264-9.

48. Choi G, Kang HY, Modi HN, et al. Risk of developing seizure after percutaneous endoscopic lumbar discectomy. J Spinal Disord Tech 2011;24:83-92.

49. Yeung AT, Tsou PM. Posterolateral endoscopic excision for lumbar disc herniation: surgical technique, outcome, and complications in 307 consecutive cases. Spine (Phila Pa 1976) 2002;27:722-31. 